\title{
Montaigne und Paracelsus
}

\author{
Ein Essay von René Bernoulli
}

\section{ZUSAMMENFASSUNG}

In diesem Jahr 1992 wird an zahlreichen Kongressen und Tagungen des 400. Todestages von Michel de Montaigne gedacht (1533-1592). Ich komme der Aufforderung des Redaktors des Gesnerus gerne nach, einen Beitrag zu diesem Zentenarium zu liefern. Als Anthropologe gehört Montaigne zweifelsohne auch zur Geschichte der Medizin.

In der vorliegenden Abhandlung wird der Perigourdiner Michel de Montaigne in einzelnen seiner Auffassungen und Äusserungen dem aus Einsiedeln gebürtigen Paracelsus (Theophrastus Bombastus von Hohenheim, ca. 14931541) gegenübergestellt. Beide waren sich einig in der Kritik, die sie, jeder auf seine Art, an der Medizin ihrer Zeit ausübten. Montaigne hat mehrmals Paracelsus erwähnt. Beide sind heute noch modern; beide erleben in unserer Epoche eine Renaissance.

\section{Einleitung}

In seinem monumentalen Werk Grosse Ärzte schliesst Henry E.Sigerist das Kapitel über Paracelsus mit folgender Würdigung:

«Mit Paracelsus betritt der Norden die Bühne der Medizin. Von vornherein wild, mit ungestümer Kraft, in faustischem Drang aufs Ganze zielend. [...] Paracelsus schreibt und wird verlacht. [...] Paracelsus greift an die Grundfragen der Heilkunde, die zeitlos im Wesen des Arzttums begründet sind. Er hinterlässt keine Schule. Nur mit Mühe werden einzelne seiner Entdeckungen Allgemeingut der Medizin. Aber sein Werk, an einem Wendepunkt der abendländischen Medizin entstanden, ist heute noch vollkommen lebendig ${ }^{1}$

Schon diese Paracelsus-Passage, für deren Objektivität ein grosser Medizinhistoriker bürgt, weist auf Bezüge hin, die, adäquat verstanden, auch Michel de Montaigne, den Autor der Essais, betreffen können. Daher die direkte Herausforderung, die zwei Renaissance-Autoren einander gegenüberzustellen.

Wohl betritt mit Montaigne nicht der rauhe, gebirgige Norden alemannischer Lande, sondern der liebliche Südwesten Frankreichs, die «douce 
France» ${ }^{2}$, die Bühne der Anthropologie. Und wenn Paracelsus an die «Grundfragen der Heilkunde» greift, so hält Montaigne mit seinen Essais dem Menschen einen Spiegel vor, der in einer bis dahin unbekannten Weise Grundfakten menschlicher Existenz aufdeckt ${ }^{3}$.

Paracelsus wurde «verlacht». Montaigne machte eine ähnliche, gewiss bittere Erfahrung:

«(C) Nul a été prophète non seulement en sa maison, mais en son pays, dit l'expérience des histoires. [...] En mon climat de Gascogne, on tient pour drôlerie de me voir imprimé. D'autant que la connaissance qu'on prend de moi s'éloigne de mon gîte, j'en vaux d'autant mieux. J'achète les imprimeurs en Guyenne, ailleurs ils m'achètent» (III, ii, 808 f., pl. 728).

Und die Geistesgeschichte zeigt zur Genüge, dass beide, Paracelsus und Montaigne, oft missverstanden und ihre Werke verfälscht wurden.

Paracelsus «hinterlässt keine Schule». Auch Montaigne begründete keine Schule im traditionellen Sinn. Er bekennt:

«(B) Je n'enseigne point, je raconte» (III, ii, 806, pl. 726).

Aber Montaigne hatte seine Arbeit unterschätzt. Sein Werk besitzt nämlich eine ungeheure Wirkkraft. Über Descartes und Pascal, Francis Bacon und Shakespeare, über Rousseau und so viele andere, Emmanuel Kant nicht ausgeschlossen, übte Montaigne einen Einfluss auf die Nachwelt aus, der heute besonders spürbar ist, nicht zuletzt auch in der Medizin. Und wenn Sigerist erklärt, dass der sich auf seine Sendung besinnende Arzt in Paracelsus einen Führer findet, so zeigt die Erfahrung, dass Ärzte und medizinische Forscher immer wieder bei Montaigne Belehrung gesucht oder Rechtfertigung für ihr Tun gefunden haben. Es sei beispielsweise der Arżt François Batisse zitiert :

«Et pourtant, lorsqu'on suit chez lui [Montaigne] cette curieuse peinture des choses de la médecine, ces observations faites au gré du jour et de l'heure, et toujours avec une clarté, une précision, une solidité, une couleur saisissantes, on se rend rapidement compte qu'on peut en tirer d'utiles enseignements» ${ }^{4}$.

Paracelsus' Werk «ist heute noch vollkommen lebendig», Montaignes Essais «sind ein Weltbuch geworden» ${ }^{5}$, um ein Wort des Freiburger Romanisten Hugo Friedrich zu gebrauchen. Und wie es eine internationale und nationale Paracelsusgesellschaften gibt, so besteht eine Société Internationale des Amis de Montaigne mit Sitz in Paris. Durch ihr Bulletin wird der Kontakt zwischen den in der ganzen Welt zerstreuten Montaigne-Forschern und nationalen Korrespondenten aufrechterhalten. 
Die Konfrontation der grossen Renaissance-Gestalten Paracelsus und Montaigne wird noch interessanter und bemerkenswerter durch den Umstand, dass zentrale Themen bei beiden Forschern in ähnlicher, wenn nicht identischer Form auftreten, ja, dass ihr Reflektieren am gleichen Ursprung ansetzt. Die Gegenüberstellung wird gar zwingend durch die belegbare Tatsache, dass Paracelsus für Montaigne kein Unbekannter war, wie noch ersichtlich sein wird. Der Basler Historiker Herbert Lüthy bemerkt im Nachwort seiner Teilübersetzung der Essais, dass Montaigne im deutschen Sprachbereich nicht besonders wohlwollend behandelt wurde:

«Man kann wohl sagen, dass Montaigne im deutschen Sprachbereich seit 1800 schlecht behandelt worden ist - und doch hätte ein Hauch Montaignescher Skepsis und Wahrhaftigkeit dem deutschen Geist sicherlich nur gut tun können» ${ }^{6}$.

Die Erfahrung zeigt tatsächlich, dass Montaigne trotz seiner überragenden Bedeutung vielerorts bestenfalls als «Erfinder des leichten Essais» bekannt ist, der «wenigstens als geistreicher und liebenswürdiger Plauderer geschätzt» ${ }^{7}$ wird.

Schier unausrottbare Legenden - auch im französischen Sprachbereich bieten ein schiefes Bild seiner Persönlichkeit und seines Werkes. Angesichts der oft etwas zweifelhaften Montaigne-Rezeption ist es im Hinblick auf unser Thema unerlässlich, einige Daten anzuführen.

\section{Michel de Montaigne und sein Werk}

Montaigne entstammt einer alten Kaufmannsfamilie aus Bordeaux, namens Eyquem. Sein Urgrossvater, im Grosshandel mit Wein, gesalzenen Fischen und Pastellfarben reich geworden, kaufte 1477 das Schloss Montaigne. Erst sein Enkel, der Vater unseres Philosophen und Bürgermeister von Bordeaux, begann den Titel «Seigneur de Montaigne» zu führen. Die Adelsprädikate der Herren von Hohenheim und der «Seigneurs de Montaigne» dürften sich etwa gleichwertig sein.

Michel de Montaigne wurde am letzten Februartag des Jahres 1533 nach neuer Zeitrechnung geboren. Er erhielt eine gut gemeinte, psychohygienisch jedoch eher fragwürdige, erste Erziehung. Über seine Universitätsstudien sind wir nicht besonders gut orientiert. Doch dürfte feststehen, dass er in Paris u.a. bei Jacques Dubois medizinischen Unterricht genoss. Dieser Mediziner, der in der Medizingeschichte unter dem Namen Sylvius besser 
bekannt ist (1478-1555), hatte das seltene Glück, unter seinen Schülern später hervorragende Gelehrte zu haben, so ausser Montaigne Andreas Vesal, Michel Servet, Conrad Gessner und andere. Sylvius blieb trotz der durch seinen Schüler Vesal ausgelösten Revolution in der Anatomie jedoch überzeugter Galenist.

Nach Abschluss seiner Studien ergriff Montaigne die Karriere eines Magistraten. Im Jahre 1571 entsagte er aus Gründen, die noch nicht restlos geklärt sind, allen Ämtern und zog sich auf sein Schloss zurück, um sich wie er sich ausdrückt - «am Busen der gelehrten Jungfrauen ganz dem Studium zu widmen» ${ }^{8}$.

Ein erstes Ergebnis lag 1580 vor : in diesem Jahr erschienen in Bordeaux die zwei ersten Bücher seines Hauptwerkes Les Essais. Schon zwei Jahre später, 1582, kam die etwas erweiterte zweite Auflage heraus; 1587, aber diesmal in Paris, eine weitere Auflage, vielleicht ein Raubdruck; und 1588, ebenfalls in Paris, die um ein drittes Buch vermehrte sogenannte fünfte Edition.

Von dieser Ausgabe 1588 besass Montaigne ein Exemplar, an dessen Text er in den vier Jahren, die zu leben ihm noch vergönnt waren - Montaigne starb am 13. September 1592 - unermüdlich weiter arbeitete. In diesem Buch brachte er zahllose handschriftliche Einschübe und Zusätze an, strich Sätze und ersetzte sie gegebenenfalls durch andere - kurzum, er war mit der Überarbeitung seines Textes bis an sein Lebensende vollauf beschäftigt. Dieses Arbeitsexemplar hat sich erhalten; es befindet sich in der Bibliothèque Municipale von Bordeaux, daher seine Bezeichnung Manuscrit (oder Exemplaire) de Bordeaux.

Montaigne schrieb sein Werk - mit Ausnahme der zahlreichen, vorwiegend lateinischen Zitate - in französischer Sprache. Vor den Essais war nur eine kleine philosophische Arbeit, die 1555 erschienene Dialectique des Petrus Ramus, in französischer Sprache gedruckt worden. Gewiss entsprach Montaignes Schreibweise noch nicht dem klassischen Französisch des 17. Jahrhunderts. Auf Montaignes Stil trifft zu, was Sigerist (a. a. O.) über die Schreibweise des Paracelsus sagt:

«Er schreibt, wie er denkt, in seiner Muttersprache. Sie ist noch spröde, und es fällt oft schwer, sich in ihr auszudrücken.»

«(A) [...] c'est aux paroles à servir et à suivre, et que le Gascon y arrive, si le Français n'y peut aller» (I, xxvi, 171, pl.135),

bekennt Montaigne, sich seiner nicht immer ganz runden Sprachweise bewusst. 
«(A) Le parler que j'aime, c'est un parler simple succulent et nerveux, court et serré [...] éloigné d'affectation $[\ldots]$ décousu et hardi; chaque lopin y fasse son corps» (I, xxvi, $171 \mathrm{f}$., pl.135).

Und wenn Sigerist meint, nur der Deutsche könne Paracelsus ganz verstehen, so ist darauf hinzuweisen, dass es zum Verständnis Montaignes eines Quentchens «esprit gaulois» bedarf. Montaignes eigenwilliger Stil ist schuld, dass es schier unmöglich ist, die Essais adäquat ins Deutsche zu übersetzen ${ }^{9}$.

Montaigne gab seinem Werk den Titel Essais. Überlassen wir es den Literaturhistorikern zu entscheiden, ob Montaigne der Schöpfer der literarischen Gattung des Essays ist. Wir aber haben uns zu fragen, was er unter dem Begriff «essai» verstand. Das Wort essai geht etymologisch auf den lateinischen Terminus exagium zurück. Dieser Ausdruck findet sich allerdings nicht bei den Klassikern, denn er gehörte zur Sprache des Volkmarktes. Er bezeichnete zunächst das Gewicht und das Wägen, im besonderen das examen trutinae, das genaue Beobachten des Züngleins am Waagebalken. Daraus im erweiterten Sinn ein jedes examen quodvis diligens et accuratum. Exagium hängt mit dem Tätigkeitswort exigere zusammen, das primär «verlangen», «fordern» bedeutet, dann im übertragenen Sinn «untersuchen», «prüfen», «abwägen». Der Terminus exagium beinhaltet somit die Tätigkeit des Prüfens. Exagium verweist auf ein beobachtendes, empirisches Geschehen, auf die prüfende Untersuchung, auf die Erfahrung, und diese Sinninhalte hatte im 16. Jahrhundert das von ihm abstammende Wort essai : exagium - exaium - essaium - essai.

In dieser Sinndeutung wollte Montaigne den Titel seines Werkes verstanden wissen. Wohl wurde Montaigne auch schon unter die Moralisten eingereiht, doch darf diese Beurteilung nicht so verstanden werden, als habe er primär dem Menschen bessere Sitten beibringen wollen - was er auch tat sondern vielmehr im ursprünglichen Sinne des Schilderers von mores, vom menschlichen Verhalten, Benehmen und seinem Charakter.

Anders formuliert : den faktischen Menschen darzustellen war sein Anliegen :

«(A) Car en l'étude que je fais, duquel le sujet c'est l'homme» (II, xvii, 634, pl.566); «(B) Les autres forment l'homme, je le récite» (III, ii, 804, pl. 724).

Und das zur Verwirklichung seiner Absicht dienende Instrument: das «essai», die Beobachtung, die Erfahrung. 


\section{Empirie und Skepsis}

Mit der Empirie begegnen wir einer grundlegenden philosophischen Haltung, die Paracelsus mit Montaigne verbindet.

«die medizin ist nichts als ein grosse gewisse erfahrenheit, nemlich das alles, so der tut, in der experienz stet. [...] und welcher sein sachen nit mit der experienz gelernt hat, und mit der warheit, die in ir ist, derselbig ist ein zweifelhaftiger arzt, und was die experienz, die als ein richter ist, bewert oder nit bewert, das sol abgenomen oder nit werden» ${ }^{10}$.

Erfahrung und Zweifel bilden eine methodologische Dualität. Wäre Paracelsus nicht Skeptiker gewesen, so hätte er wie seine Kollegen nach der damals üblichen galenischen Lehre handwerksmässig seinen Arztberuf ausgeübt. Methodologisch geht die Skepsis der Empirie voraus. Und Paracelsus gehört zu den grossen Skeptikern:

«Wie kann der Mensch sagen : «ich bin gewiss», wo ihm so viel dazu fehlt? Wahr ist vielmehr, dass er um nichts weiss» ${ }^{11}$.

Montaigne ist, wo möglich, der noch grössere Skeptiker als Paracelsus. Paracelsus weiss, ähnlich der traditionellen sokratischen Skepsis, wenigstens das eine: dass er nichts weiss. Montaigne weiss nicht einmal dieses. Denn es könnte sein, dass unter den «(B) cent et cent contraires partis» (II, xii, 504, pl.446), die die Philosophie uns darbietet, doch ein Philosophem die Wahrheit enthielte. Aber wie diese eine richtige Lehre aus der Unzahl der vorliegenden Theorien erkennen?

«(C) Qu'irai-je choisir?» (II, xii, 504, pl.446), fragt Montaigne. «(B) Vaut-il pas mieux demeurer en suspens que de s'infrasquer (sich verirren) en tant d'erreurs que l'humaine fantasie a produite? Vaut-il pas mieux suspendre sa persuasion (Überzeugung) que de se mêler à ces divisions séditieuses et querelleuses?» (II, xii, 504, pl.446).

Fragen, die auch den modernen Arzt noch zu beschäftigen vermögen ${ }^{12}$.

Die Skepsis als Methode bildet den Ursprung des Reflektierens bei Paracelsus wie bei Montaigne. Diese grundlegende Skepsis erweist sich auch wirksam in bezug auf die Einstellung gegen die Gelehrten ihrer Zeit:

Paracelsus: «etwan ist vermeint worden, dass Mompelier das Tor der Arznei sei und auch Salern. Und ist doch nichts von ihnen gebracht worden, als allein ein rots barettlein» ${ }^{13}$.

D.h.: Paracelsus vermisst bei seinen Kollegen ein eigenständiges geistiges Schaffen.

Montaigne: «(C) Tout fourmille de commentaires, d'auteurs il est grande cherté» (III, xiii, 1069 , pl. 968. 
Daher sein hartes Urteil über die Erziehungs- und Ausbildungsmethoden seiner Zeit; sie erzeugen nur «(A) des ânes chargés de livres» (I, xxvi, 177, pl.141).

Es wäre jedoch falsch, Paracelsus oder Montaigne unterschieben zu wollen, ihre Skepsis führe zu einem existentiellen Nihilismus. Im Gegenteil:

«Wir sind zum Wachen geboren, nicht zum Schlafen!» ${ }^{14}$, ruft Paracelsus schon im ersten Buch seiner Fünf Bücher von den unsichtbaren Krankheiten aus.

Montaigne proklamiert: «(A) Nous sommes nés pour agir» ( $\mathrm{I}, \mathrm{xx}, 89$, pl.69). «(C) Et estre consiste en mouvement et action» (II, viii, 386, pl. 325).

Es ist darauf hinzuweisen, dass der methodologische Skeptizismus weder bei Paracelsus noch bei Montaigne zu religiöser Gleichgültigkeit führte. Beide waren gläubige Menschen.

Die Gegenüberstellung von Paracelsus-Texten mit Essais-Zitaten liesse sich fortsetzen, wobei auch divergente Stellen anzutreffen wären. Doch wenden wir uns der Paracelsus-Rezeption in den Essais zu.

\section{Paracelsus in den Essais}

Eine erste Paracelsus betreffende Stelle findet sich im 12. Kapitel des 2. Buches. Dieses bei weitem grösste Kapitel der ganzen Essais, dem Montaigne den Titel «Apologie de Raymond Sebon» gab, gehört zu den umstrittensten Abschnitten des Werkes. Es dürfte allerdings jenen Montanisten beizupflichten sein, die in diesem Kapitel das Kernstück des ganzen Buches sehen. In ihm finden sich jene eindrücklichen Ausführungen, in denen Montaigne die Fragwürdigkeit der natürlichen Erkenntnismöglichkeiten des Menschen aufdeckt. Die «Apologie» gehört zweifelsohne zu den philosophisch und weltanschaulich wichtigsten Kapiteln der Essais.

Nun gibt es nach Paracelsus keine echte Medizin ohne philosophische Grundlegung:

«Schlimm ist es um einen Arzt bestellt, der sich ein Arzt nennt und nichts versteht von der Philosophie» ${ }^{15}$.

Infolge der zwischen Medizin und Philosophie bestehenden Beziehungen überrascht es nicht, wenn Montaigne auch die Medizin in den methodologischen Zweifel miteinbezieht. Dabei kommt er auf Paracelsus zu sprechen. 
«(A) Ainsi, quand il se présente à nous quelque doctrine nouvelle, nous avons grande occasion de nous en défier, et de considérer qu'avant qu'elle fut produite, sa contraire était en vogue; et, comme elle a été renversée par celle-ci, il pourra naître à l'avenir une tierce invention qui choquera de même la seconde. Avant que les principes qu'Aristote a introduits fussent en crédit, d'autres principes contentaient la raison humaine, comme ceux-ci nous contentent à cette heure. Quelles lettres ont ceux-ci, quel privilège particulier, que le cours de notre invention s'arrête à eux, et qu'à eux appartient pour tout le temps à venir la possession de notre créance? ils ne sont non plus exempts du boute-hors qu'étaient leurs devanciers. [...] Combien y a-t-il que la médecine est au monde? On dit qu'un nouveau venu, qu'on nomme Paracelse, change et renverse tout l'ordre des règles anciennes, et maintient que jusques à cette heure, elle n'a servi qu'à faire mourir les hommes. Je crois qu'il vérifiera aisément cela; mais de mettre ma vie à la preuve de sa nouvelle expérience, je trouve que ce ne serait pas grand'sagesse.» (II, xii, $570 \mathrm{f} .$, pl. $504 \mathrm{f}$.)

Dieser Abschnitt findet sich schon in der Urausgabe von 1580, d.h. Montaigne kannte Paracelsus schon verhältnismässig früh. Er wusste, dass Paracelsus zu jenen Ausnahmegestalten zählte, die als revolutionär eingestellte Ärzte die Ohnmacht der damaligen Medizin aufzeigten. Aber - und hier spricht Montaignes radikale Skepsis - sich Paracelsus vorbehaltlos anzuvertrauen, wäre wahrscheinlich doch nicht allzu klug, denn auch Paracelsus wird wohl später von anderen in Frage gestellt werden. Immerhin, Montaigne greift Paracelsus nicht an, was bei seiner Skepsis gegenüber Medizin und Ärzten einem durchaus positiven Urteil gleichkommt.

Zwei weitere Paracelsus-Stellen finden sich im 37. Kapitel des 2. Buches, dem Montaigne den Titel «De la ressemblance des enfants aux pères» gab. In ihm lässt Montaigne seiner Skepsis in bezug auf die Medizin seiner Zeit und den Dünkel der Ärzte freien Lauf:

\begin{abstract}
«(A) C'est une précieuse chose que la santé, et la seule qui mérite qu'on y emploie, non le temps seulement, la sueur, la peine, les biens, mais encore la vie à sa poursuite. [...] Toute voie qui nous mènerait à la santé, ne se peut dire pour moi ni âpre, ni (C) chère. (A) Mais j'ai quelques autres apparences (Gründe) qui me font étrangement défier de toute cette marchandise. Je ne dis pas qu'il n'y en puisse avoir quelque art; qu'il n'y ait, parmi tant d'ouvrages de nature, des choses propres à la conservation de notre santé, cela est certain. Mais je dis, ce qui s'en voit en pratique, il y a grand danger que ce soit pure imposture, j'en crois leurs confrères Fioravant et Paracelse» (II, xxxvii, 765, pl. 685 f.)
\end{abstract}

Dieser Text gehört zu jenen, die in ganz besonderer Weise für Montaignes fortwährende Aktualität zeugen. Selbst die Frage nach den Kosten für die Wiederherstellung der Gesundheit, ein gewiss zeitgemässes Thema, findet sich angedeutet. Diesbezüglich scheint das Geld für Montaigne keine Rolle zu spielen - «[...] ni chère»! Dass Montaigne gerade an dieser Stelle sich des im Ursprung seines Denkens immer zeitgemässen Paracelsus erinnert, 
spricht für den Eindruck, den Hohenheim auf ihn gemacht hatte. Nebenbei bemerkt: Leonardo Fioravanti war ein in der Mitte des 16. Jahrhunderts in Bologna wirkender Arzt und einer der wenigen italienischen ParacelsusAnhänger.

Obiges Zitat findet sich, wie erwähnt, bereits in der ersten Ausgabe der Essais. Für die Edition von 1588 streicht Montaigne jedoch den letzten Paracelsus und Fioravanti betreffenden Satz. In der Zwischenzeit hatte sich in Paris nämlich eine dem Ruf Hohenheims schadende Begebenheit zugetragen, von der Montaigne zweifelsohne Kenntnis erhalten hatte. Ein Kurpfuscher hatte als «disciple de Paracelse» viel von sich reden gemacht. Sogar das Parlament verlangte eine Untersuchung; vor dem Untersuchungsausschuss der Medizinischen Fakultät versagte der angebliche Paracelsus-Jünger ganz und gar. Bei der damaligen, für einen Franzosen nur beschränkten Möglichkeit, ein eingehendes Paracelsus-Studium zu betreiben, musste ein derartiges Vorkommnis unweigerlich zu einer vorsichtigeren Einschätzung Hohenheims führen.

Die dritte Paracelsus-Stelle der Essais findet sich ebenfalls im Kapitel II, xxxvii. Nach einem Überblick über die Medizingeschichte und die sich immer wieder ablösenden und widersprechenden Ansichten fährt Montaigne fort:

«(A) Depuis ces anciennes mutations de la médecine, il y a eu infinies autres jusques à nous, et le plus souvent mutations entières et universelles, comme sont celles que produisent de notre temps Paracelse, Fioravanti et Argenterius [Argenterius, aus dem Piemont gebürtig, war ein besonders heftiger Antigalenist] : car ils ne changent pas seulement une recette, mais à ce qu'on me dit, toute la contexture et police du corps de médecine, accusant d'ignorance et de piperie (Betrug) ceux qui ont fait profession jusque à eux. Je vous laisse à penser où en est le pauvre patient!» (II, xxxvii, 772, pl.693.)

Es ist sicher nicht übertrieben zu behaupten, diese Stelle zeuge von einer recht guten Paracelsus-Kenntnis. Hierbei ist allerdings zu beachten, dass, trotz der Übereinstimmung mit Paracelsus in manchen medizinischen Ansichten, Montaigne kein Epigone Hohenheims war. Die Quellen, aus denen ihre jeweilige Geisteshaltung entspringt, sind allzu verschieden. So stellt Paracelsus apodiktisch fest:

«Dan es ist ein grosser irsal und stets übel, das so vil [...] arzte [...] nämlich zu Mompelier, Salerna, Paris, die da wöllen vor allen den kranz [Ehre] haben und jedermann verachten und doch selbst nichts wissen, noch können» ${ }^{16}$.

Und polternd fährt Paracelsus fort: 
«ir müsset mir nach mit euerem Avicenna, Galeno [...] und ich nit euch nach; ir mir nach, ir von Paris, von Montpelier, von Salern, von Wien, von Cöln, von Wittenberg und all ir in der summa, und keiner muss ausgenommen sein, im hindersten badwinkel nicht bleiben» ${ }^{17}$.

Einen solch selbstherrlichen Ton hat Montaigne nie angeschlagen; er wäre erheblich vorsichtiger gewesen. Es sei an dieser Stelle daran erinnert, dass Paracelsus im Februar 1528, in Nacht und Nebel, die Stadt Basel, wo er kaum ein Jahr zuvor als Stadtarzt angestellt worden war, fluchtartig verliess. Er hatte den Ton mit Behörden und Kollegen nicht gefunden, im Gegensatz zu Montaigne, der beispielsweise zweimal Bürgermeister von Bordeaux war.

Es sei abschliessend noch die Frage aufgeworfen, woher Montaigne seine Paracelsus-Kenntnis hatte. Eines dürfte feststehen: er konnte Paracelsus nicht aus Originalwerken kennen, da er, nach eigenem Geständnis, der deutschen Sprache nicht mächtig war. Doch gab es schon vor 1572 eine Anzahl lateinischer und französischer Übersetzungen von Paracelsus-Texten. Montaigne besass eine Bibliothek, auf die er mit Recht stolz war - «(A) Ma librairie, qui est des belles entre les librairies de village» (II, xvii, 650, pl.580); sie enthielt nach seinen Angaben um die tausend Bände. Von diesen Werken konnte bis jetzt rund ein Drittel mit Sicherheit identifiziert werden. Ohne Zweifel ist die eine oder andere Paracelsus-Studie in Übersetzung hier einzureihen.

Nach Karl Jaspers ist Philosophie das über alle Räume und Zeiten hinweg sich erstreckende Gespräch unter den Grossen des Geistes. In dieser Runde waren wir lauschend anwesend. Waren wir aufmerksam? Ertönte da nicht der die je eigene Existenz zentral treffende Aufruf: «Alterius non sit, qui suus esse potest»? ${ }^{18}$ Unter allen am philosophischen Gespräch unmittelbar Beteiligten hat wohl keiner wie Michel de Montaigne diesen Appell aufgenommen und in sein Denken und Handeln mit dem ihm eigenen realistischen Optimismus integriert.

«(A) Certes l'homme d'entendement n'a rien perdu s'il a soi-même» (I, xxxix, 240, pl.206).

«(B) Nous sommes chacun plus riche que nous ne pensons.» (III, xii, 1038, pl.943). 


\section{Anmerkungen}

1 Henry E.Sigerist, Grosse Ärzte. Eine Geschichte der Heilkunde in Lebensbildern, 6. Aufl., München, Lehmann, 1970, S. 97 (1. Aufl. 1932). - In den folgenden Ausführungen wird mehrfach auf Auszüge aus dem - leicht gekürzt - zitierten Abschnitt zurückgegriffen.

2 Schon in der Chanson de Roland wird das Frankenreich an zahlreichen Stellen als «dulce France» bezeichnet $(\mathrm{u}=\mathrm{ou})$.

3 Montaigne arbeitete an seinen Essais vom Jahre 1572 bis zu seinem Tode am 13. September 1592. Die erste Ausgabe erschien schon 1580, die Ausgabe letzter Hand 1588. In einem Exemplar dieser Edition brachte er zahllose Korrekturen und Zusätze an. Dieses kostbare Exemplar hat sich als Manuscrit (Exemplaire) de Bordeaux in der Bibliothèque Municipale dieser Stadt erhalten. Ich habe 1978 eine Faksimileausgabe dieses Exemplars in Genf und Paris bei Slatkine herausgegeben. - In den folgenden Montaigne-Zitaten in modernerer Orthographie bezeichnet die erste römische Zahl (Majuskel) das Buch, die zweite (Minuskel) das Kapitel der Essais; die erste arabische Zahl die Seite der Essais-Edition von P. Villey/V.L. Saulnier, Lausanne, La Guilde du Livre, 1965; die letzte Angabe «pl.» mit arabischen Ziffern die Tafel (planche) meiner Faksimileausgabe. Die in Klammern gesetzten Buchstaben geben die Edition an, in der der folgende Text zum erstenmal erschien, und zwar (A) die Ausgabe 1580, (B) 1588 und (C) den handschriftlichen Eintrag im Manuscrit de Bordeaux. Diese Angaben sind für ein tieferes Verständnis des Montaigne-Textes oft unerlässlich.

4 François Batisse, Montaigne et la Médecine, Paris, Les Belles Lettres, 1962, S. 7.

5 Hugo Friedrich, Montaigne, zweite neubearbeitete Auflage, Bern und München, Francke, 1967, S.9.

6 Herbert Lüthy, Michel de Montaigne, Essais, Auswahl und Übersetzung, Zürich, Manesse Verlag, 1953, S. 888.

7 Karl Vorländer, Geschichte der Philosophie, Bd.I, Leipzig, Felix Meiner, 1913, S. 318.

8 Nach einer im Eingangsturm zu seinem Schloss, wo sich sein Studierzimmer befand, angebrachten lateinischen Inschrift, siehe Léonie Gardeau und Jacques de Feytaud, Le château de Montaigne. Société des Amis de Montaigne, Paris, 1971.

9 Die zweifellos beste deutsche Übersetzung der Essais ist dem emeritierten Basler Professor für Geschichte Herbert Lüthy zu verdanken, Michel de Montaigne, Essais, Auswahl und Übersetzung, Manesse, 1953. - Leider sind nur zwei Drittel des Montaigne-Textes übersetzt.

10 Zitiert nach Hans Fischer, Anthropologie des Paracelsus in: Arzt und Humanismus, Zürich und Stuttgart, Artemis, 1962, S. 196.

11 Robert Blaser, Himmel und Erde machen den Menschen, Salzburg, Residenz, 1958, S. 22.

12 Zu der hier angeschnittenen Thematik siehe meine Untersuchung, «Que sais-je ? Contribution à l'étude de la gnoséologie de Montaigne», Bulletin de la Société des Amis de Montaigne, $5^{\circ}$ série, $\mathbf{n}^{\circ} 16,1975$, S. 15-36.

13 Ernst Kaiser, Paracelsus, Rowohlt Taschenbuch, 1969, S. 70.

14 R. Blaser, a. a. O., S. 36 (Morb. Invis. I/9, 256).

15 R. Blaser, a. a. O., S.67 (Opus, I/9, 122.)

16 E. Kaiser, a.a. $0 .$, S. $71(\mathrm{I} / 9,139$.

17 E. Kaiser, a.a. O., S. $72(\mathrm{I} / 8,137$.

18 Hohenheims bekannte Lebensmaxime. 


\section{Summary}

\section{Montaigne and Paracelsus}

After some general remarks on Michel de Montaigne (1533-1592), who died just 400 years ago, the author compares him with another most original thinker of the $16^{\text {th }}$ century: Paracelsus. Montaigne and Paracelsus were akin in their fundamental scepticism about human knowledge and convictions. Speaking, in his "Essais", of medicine Montaigne repeatedly quotes Paracelsus. These quotations show that Montaigne had some knowledge of Paracelsus' writings and that, to some extent, he was in sympathy with his ideas.

\section{Résumé}

\section{Montaigne et Paracelse}

Michel de Montaigne, dont le monde intellectuel commémore cette année 1992 le quatrième centenaire de son décès survenu le 13 septembre, mérite que les historiens de la médecine ne laissent pas passer cet événement sous silence. En effet, Montaigne appartient non seulement à l'histoire de la pensée philosophique, mais aussi à l'histoire de la médecine. Dans la présente étude certaines idées de Montaigne touchant de près la médecine ont été mises en relief avec les pensées de Paracelse qui, lui aussi, fut un révolutionnaire dont les conceptions médicales devancèrent son époque.

Avis aux Amis de Montaigne

Notre confrère, le docteur Philippe Albou, a récemment publié dans le périodique hebdomadaire Impact médical du 29 mai 1992 un intéressant article, «Les médecins à l'essai», enrichi de deux belles gravures en couleur, l'une représentant Montaigne, l'autre Paracelse; le personnage principal est Montaigne.

Nous devons au même auteur un spirituel essai, bien documenté, Une Interview de Montaigne sur la médecine, disponible à la librairie Klincksieck, 11 rue de Lille, F-75007 Paris.

Dr. med. et phil. René Bernoulli

Ehrendozent der Med. Fakultät

Amselstrasse 35

4059 Basel 